\title{
Investigating the Synergistic Relationship between Enterprise Resource Planning and Business Intelligence
}

\section{Nazeen Ansari*, Gopakumaran Thampi}

St. Francis Institute of Technology, Department of Information Technology, Mumbai-400103, India Thadomal Shahani Engineering College, Department of Information Technology, Mumbai-400050, India

\section{ABSTRACT}

This study aims to discuss the synergistic relationship between enterprise resource planning (ERP) and business intelligence (BI). It focuses on the benefits obtained from ERP and $\mathrm{BI}$ framework integration. The purpose of this study is to identify the software engineering challenges associated with ERP-BI integration. It also discusses methods for ERP-BI integration and shift toward open source and standard-based integration technologies.

\section{KEYWORDS:}

Integration; Software engineering challenges; ERP-BI.

\section{Council for Innovative Research}

Peer Review Research Publishing System

Journal: INTERNATION JOURNAL OF COMPUTERS AND TECHNOLOGY

Vol. 13, No. 10

editorijctonline@gmail.com

www.ijctonline.com, www.cirworld.com 


\section{INTRODUCTION}

Software systems play a crucial role in an organization as they integrate key information present within the organization. Many organizations spend loads of money on such systems. To obtain returns on such investments, these systems must be used for many years. Depending on the organization size, the software system lifespan varies. Software engineering is a discipline that integrates methods, tools, and procedures for developing computer software. Enterprise resource planning (ERP) systems can be considered as one of the most innovative developments in information technology since 1990s.

Nowadays, many software systems are available in the market aiming to increase an organization's productivity and efficiency. The desire to integrate such systems turns out to be a priority for business managers [1]. Reducing the effort required for software integration is on a rise in organizations [2].

\section{ENTERPRISE RESOURCE PLANNING}

ERP is a software architecture that enables information flow among different functions within an enterprise. Moreover, it enables information sharing through organizational units and geographical locations. It enables decision makers to have an enterprise-wide view of the information they require in a timely, reliable, and consistent manner.

Lately, buying a business application that includes transaction processing systems for tasks such as accounting, manufacturing, or human resources as a packaged product has been possible. Packages that perform such operation are referred to as ERP systems [3].

ERP is a set of integrated business applications or modules that perform common business functions such as general ledger, accounting, or order management. ERP systems are the backbone for most organizations that integrate backoffice applications such as finance, purchasing, HR, and inventory management.

ERP integrates an organization's business department functionalities into a single system in order to meet the department requirements and share information easily [4].

ERP systems are independent systems that contain modules such as finance, inventory, production planning, and HR. Progressively, technologies such as supply chain management (SCM), customer relationship management (CRM), product information management, and business intelligence $(\mathrm{BI})$ came into existence and can be interfaced with ERP systems [5].

\section{BUSINESS INTELLIGENCE}

According to the Gartner Group (1996), BI is defined as the application of a set of methodologies and technologies, such as J2EE, .Net, web services, XML, data warehouse (DW), online analytical processing (OLAP), and data mining (DM), to improve enterprise operation effectiveness, support management, or decision to achieve competitive advantages.

To improve decision making efficiency, $\mathrm{BI}$ explores several technological tools, produces reports, and forecasts, which includes DW; extract, transform, and load (ETL); OLAP; DM; text mining; web mining; data visualization; geographic information systems; and web portals [6].

BI can be classified into three types: (i) data capture/acquisition, (ii) data storage, and (iii) data access and analysis. Data is collected from both internal and external data sources. Internal data sources are an organization's operational database and DW, whereas external data sources include data from customers, suppliers, government agencies, competitors, and internet. The collected data is stored in DW after the ETL process. Finally, the stored data is analyzed for decision making [6].

ETL solutions available in the market, for example, SAS Q, DataStage EX, Oracle Warehouse Builder, DataMirror Transformation Server, Microsoft Data Transformation Services, differ by inter alia, access to data, data processing algorithms, components used to filter and search data [7].

Sectors using $\mathrm{BI}$ systems include manufacturers, e-commence businesses, telecommunication providers, airlines, retailers, health systems, financial services, and bioinformatics. Hotels use BI systems for customer support, market research, segmenting, product profitability, inventory and distribution analysis, statistical analysis, multidimensional reports, fraud detection, etc. Vendors such as Cognos, Information Builders, and SAS are working with Google to use the Google OneBox with real-time BI systems [8].

\section{NEED FOR INTEGRATING ENTERPRISE RESOURCE PLANNING WITH BUSINESS INTELLIGENCE}

Restricted standard reports in applications such as ERP, CRM, and SCM-time-consuming scripting for additional reports. Availability of only static reports-no analysis possible.

For analysis and intelligence, export to Microsoft Excel—chances of data corruption and manipulation.

Organizations recognize the affluence of information within ERP systems, the challenge lies in the ways of mining them.

Since ERP systems were not initially designed to provide real-time reports to massive users, the entire system was unable to facilitate the decision support function. Progressively, organizations are focusing on $\mathrm{BI}$ tools that create data collected by ERP, CRM, and other important data-intensive applications. BI systems can pull the data in ERP systems and then perform analyses and deliver superior reporting, which help users for making timely and accurate decisions. 


\section{ENTERPRISE APPLICATION INTEGRATION}

Enterprise application integration (EAI) is an emerging integration software group that addresses more effectively the need to integrate both intra and interorganizational systems. It incorporates functionality from dissimilar applications, and combines traditional integration technologies (e.g., database-oriented middleware) with new EAl technologies (e.g., adapters and message brokers) to support efficient incorporation of information systems. Thus, EAI results in supporting data and objects, and it manages incorporation as well as custom applications, packaged systems, and e-business solution integration [9].

EAl is defined as the combination of platforms, business processes, standards, and applications that result in the continuous integration of two or more enterprise systems. EAl tools are platform and language independent, though certain tools have superior capabilities for certain technologies (e.g., .Net and J2EE) and domains (e.g., finance, healthcare, manufacturing, insurance, and public sector). Adapter development can be the most complex part of any integration solution. Adapters allow data extraction and loading from individual applications and databases to be integrated. Building adapters requires the use of an application's existing API's or API's provided by the EAI tools. EAI tools such as IBM MQ/WBI, webMethods, and TIBCO provide a set of adapters for packaged and legacy applications or databases. Software connectors are "specialized" software components that facilitate interactions among "application" software components.

\section{BENEFITS OF INTEGRATING ENTERPRISE RESOURCE PLANNING WITH BUSINESS INTELLIGENCE}

When compared to individual purchasing of ERP and $\mathrm{BI}$ systems, a combined application is a more profitable option, making such systems affordable to even low-end small and medium businesses and small and medium enterprises.

In the fast-paced nature of markets, quick and accurate decision making can either make or break an organization. Integration of these solutions automates the collection, storage, retrieval, and sorting of relevant data, and analyzing such data helps to provide meaningful and applicable reports.

The integration of $\mathrm{BI}$ and ERP systems can strengthen corporate decision-making capability by utilizing the analytical capability of $\mathrm{BI}$ system and data managerial capability of ERP system.

$>$ Integration enables finance personnel to generate revenue or expense reports rapidly.

$>$ Integration allows the controller to recognize corporate cash flow in real time.

$>$ Integration improves the profitability by analyzing transactions data and forecasting business trends.

$>$ Integration improves relations through in-depth sales data mining.

$>$ Integration reduces time to generate regular reports.

\section{METHODS FOR INTEGRATING ENTERPRISE RESOURCE PLANNING AND BUSINESS INTELLIGENCE}

1. Remote function calls. The ERP and BI software invoke each other's callable functions remotely. Calling software is usually developed by third-party vendors.

2. Third-party connectors. Connectors are built by third-party vendors that directly connect packaged front-end and backend systems.

3. Passive middleware. ERP and $\mathrm{BI}$ are connected at passive middleware level that runs on the top of the operating system. This solution functions as long as users stick to generic ERP and $\mathrm{BI}$, and they do not try to customize their processes.

4. Customized EAI. An environment of standards, platforms, and connector software that together supports enterprise integration between ERP and BI.

\section{CHALLENGES}

Due to limited interfacing by the ERP system and ERP vendors, integrating ERP applications with the outside world has been a challenge. A challenge of using dissimilar models is maintaining consistent information between them. Challenges are how to deal with components, connectors, semantics or topology, data integration, and mapping. Data and processes between dissimilar software systems, communicating data back and forth in real time or near real time is a major challenge.

\section{OPEN SOURCE AND STANDARD-BASED INTEGRATION}

Open source has emerged to be one of the transformational IT trends. Open source software (OSS) is a program for which the source code is available. OSS resulted in low total cost of software ownership for adopters, and it also created a major disruption in the traditional software space.

Excluding better performance, reliability, scalability, security, support, and a clearly defined upgrade path and identification, open source reduces the application cost by $50 \%-70 \%$ (across hardware, implementation, upgrades, administration, and support) over proprietary technologies. 
Another remarkable factor supporting the growing OSS adoption is the ability to integrate easily with external systems compared to proprietary technologies. Other benefits include faster turnaround time in gathering business requirements, adaptable to business changes, better risk mitigation, and freedom from vendor lock-in and licensing and flexibility.

There has been a noticeable shift toward open source and standard-based integration technologies. Vendor monopolies on integration technologies resulted in expensive solutions, vendor lock-ins, and proprietary technologies. Open source and standard-based solutions mean an increase in development times, but the solutions are lightweight, cheaper, and ensure no vendor lock-in. Open source technologies such as JBOSS ESB/messaging, JBPM, Mule, and JRules and standard technologies such as ML/XSLT/XPATH, JBI, and JMS to name a few.

\section{CONCLUSION}

Companies worldwide invest in enterprise information system software such as ERP, SCM, CRM, and other BI software to streamline their business processes and maximize their productivity. What ERP and other software vendors often do not address is how these dissimilar software systems would collaborate with each other, especially if these systems are from diverse vendors or run in different platforms, the value from these software systems can only be realized if they appropriately communicate with each other. By working together, ERP solutions can incorporate data from all over the organization where $\mathrm{BI}$ applications can use this consolidated data to provide a more comprehensive analysis. Hence, an integrated ERP with $\mathrm{BI}$ is an ideal way to derive effective analysis of data for effective decision making.

\section{REFERENCES}

[1] X. Larrucea, "Method engineering approach for interoperable systems development," Software Process: Improvement and Practice, vol. 13, pp. 127-133, 2008.

[2] O. Mazhelis, P. Tyrvainen, and E. Viitala, "Modelling software integration scenarios for telecommunications operations software vendors," 2007 IEEE International Conference on Industrial Engineering and Engineering Management, vol. 1-4, pp. 49-54, 2007.

[3] M. R. Imtihan, M. S. Ngadiman, and H. Haron, "An alternative model for ERP maintenance strategy," In Software Engineering, Artificial Intelligence, Networking, and Parallel/Distributed Computing, 2008. IEEE. SNPD'08. Ninth ACIS International Conference on, pp. 785-793, 2008.

[4] A. A. S. Al-Mudimigh, B. F. Saleem, and C. Z. Ullah, "Developing an integrated data mining environment in ERP-CRM model-a case study of MADAR," NAUNA International Journal of Education and Informational Technologies, vol. 2, 2009.

[5] A. Bhardwaj, "Transforming business: integrating ERP with E-business," International Journal of Advanced Research in Computer Science and Software Engineering, vol. 3, 2013. ISSN: 2277 128X.

[6] R. A. Khan and S. M. K. Quadri, "Business intelligence: an integrated approach," Business Intelligence Journal, vol. 5, pp. 64-70, 2012.

[7] C. M. Olszak and E. Ziemba, "Business intelligence systems in the holistic infrastructure development supporting decision-making in organisations," Interdisciplinary Journal of Information, Knowledge, and Management, vol. 1, pp. 47-57, 2006.

[8] B. S. Sahay and J. Ranjan, "Real time business intelligence in supply chain analytics," Information Management \& Computer Security, vol. 16, pp. 28-48, 2008.

[9] M. Themistocleous, Z. Irani, and P. E. Love, "Enterprise application integration: an emerging technology for integrating ERP and supply chains," ECIS 2002 Proceedings, vol. 88, 2002.

\section{Authors' Biography with Photo}

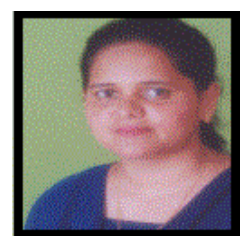

Nazeen Ansari received her Bachelor's and Master's degree in Computer Engineering and Information Technology in 1997 and 2006, respectively. Currently she is pursuing her PhD in Information Technology from the University of Mumbai. Moreover, she works as an Associate Professor at the Department of Information Technology in St. Francis Institute of Technology, Mumbai. In addition, her areas of interest include data mining, computer games, and ERP and BI. Furthermore, her research studies have been accepted and successfully published in many National and International conferences as well as Journals, especially in the areas of Computer Games and Data Mining. She is also a life time member of Computer Society of India and Indian Society for Technical Education.

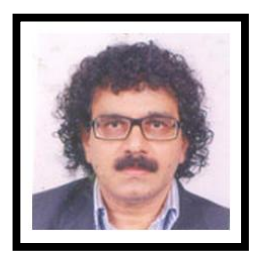

Dr. Gopakumaran Thampi received his Bachelor's and Master's degree in Mechanical Engineering in 1984 and 1996, respectively. Moreover, he completed his PhD in Technology from the University of Mumbai in 2004. He is the Principal and Professor of Thadomal Shahani Engineering College, Mumbai. He has received the Shikshak Ratan Award on October 12, 2011 in Delhi. He is a member of Research and Review committee as an expert in the subject of Information Technology of the University of Mumbai. He is also a member of Research and Review committee as an expert in the subject of Management studies of SNDT University. In addition, his areas of interest include business process reengineering in the realm of engineering education, and his areas of specialization include Integration in Enterprises. In addition, he has written three books, namely Object oriented programming in JAVA, Object oriented programming in C++, and Programming for mobile and remote computers. Furthermore, his research studies have been accepted and successfully published in many National and International conferences as well as renowned Journals. 